\title{
Avaliação radiográfica do colapso sagital do Mal de Pott
}

Radiographic evaluation of sagital kyphosis in spinal tuberculosis

Evaluación radiológica de la cifosis sagital en la tuberculosis vertebral

\author{
Carlos Humberto Targa Moreira' \\ Ricardo Shigueaki Galhego Umeta ${ }^{2}$ \\ Maria Fernanda Silber Caffaro ${ }^{3}$ \\ Robert Meves ${ }^{4}$ \\ Elcio Landim ${ }^{5}$ \\ Osmar Avanzi ${ }^{6}$
}

\section{RESUMO}

Objetivo: avaliar o colapso sagital dos pacientes com tuberculose vertebral tratados pelo modo conservador. Métodos: revisão dos prontuários de pacientes do Grupo de Cirurgia da Coluna atendidos na Santa Casa de Misericórdia de São Paulo. A deformidade em cifose foi aferida pelo método de Cobb e da cifose segmentar. Resultados: do total de 123 pacientes, $33(68,8)$ foram submetidos a tratamento conservador, sendo 17 (51\%) pacientes do sexo masculino e $16(48,8 \%)$ do sexo feminino. A idade variou de 3 a 87 anos com média de 44,1 anos. Houve aumento da cifotização local em 20 pacientes $(60,6 \%)$ e da cifose segmentar em $23(69,6 \%)$, sendo que o de pior prognóstico de progressão foi a nível toracolombar $\left(11,3^{\circ} ; p<0,05\right)$. Conclusão: nesta série de casos de tuberculose vertebral tratados sem cirurgia a evolução radiológica foi satisfatória.

\section{ABSTRACT}

Objective: to evaluate the radiographic outcomes of patients treated for spinal tuberculosis. Methods: the charts of patients of the Spine Surgery attended at the Santa Casa de Misericórdia de São Paulo were reviewed. The kyphotic deformities were measured by Cobb's method. Results: of the 123 patients, 33 (68. 8\%) were treated conservatively, 17 (51\%) were male and $16(48.8 \%)$ were female. Age ranged from 3 to 87 with a mean of 44.1 years. We noticed the increase of local kyphosis in 20 patients $(60.6 \%)$ and segmental kyphosis in $23(69.6 \%)$, with the worst prognosis of progression at the thoracolumbar $(11.3 \%)$, with a statistically significant difference $(p<0.05)$. Conclusion: the empirical therapy based on clinical diagnosis radiological showed good radiographic findings in this series of cases treated conservatively.

\section{RESUMEN}

Objetivo: evaluar los resultados radiológicos de los pacientes tratados por tuberculosis vertebral. Métodos: la revisión de los prontuarios de pacientes del Grupo de la Columna asistidos en la Santa Casa de Misericordia de São Paulo. La cifosis se mide por el método de Cobby por la cifosis segmentar. Resultados: de los 123 pacientes, 33 (el 68,8\%) fueron tratados de forma conservadora, $17(51 \%)$ pacientes eran varones y 16 $(48,8 \%)$ eran mujeres. Las edades oscilan entre 3 y 87 años, con una media de 44,1 años. Se observó el aumento de la cifosis local en 20 pacientes $(60,6 \%) y$ de la cifosis segmentaria en 23 (69,6\%), el peor pronóstico de la progresión está en la toracolombar (11,3), aunque estadisticamente significativo que diferencia $(p<0,05)$. Conclusión: el tratamiento empirico basado en clínica $X$ de diagnóstico radiológico de tórax mostró buenos resultados en esta serie de casos tratados de forma conservadora.

\footnotetext{
Trabalho realizado no Departamento de Ortopedia e Traumatologia da Faculdade de Ciências Médicas da Santa Casa de Misericórdia de São Paulo - FCMSCMSP — São Paulo (SP), Brasil. [Apresentado no XXIII Congresso De Ortopedia E Traumatologia Do Estado De São Paulo. São Paulo; 2010]

'Aluno do Curso de Aperfeiçoamento do Grupo de Cirurgia da Coluna do Departamento de Ortopedia e Traumatologia da Faculdade de Ciências Médicas da Santa Casa de São Paulo - FCMSCSP - São Paulo (SP), Brasil.

2Pós-graduando (Mestrado) do Departamento de Ortopedia e Traumatologia da Faculdade de Ciências Médicas da Santa Casa de São Paulo - FCMSCSP - São Paulo (SP), Brasil.

${ }^{3}$ Mestre; Professora Instrutora; Assistente do Grupo de Coluna do Departamento de Ortopedia e Traumatologia da Faculdade de Ciências Médicas da Santa Casa de São Paulo - FCMSCSP - São Paulo (SP), Brasil.

${ }^{4}$ Doutor; Professor Assistente e Chefe do Grupo de Coluna do Departamento de Ortopedia e Traumatologia da Faculdade de Ciências Médicas da Santa Casa de São Paulo - FCMSCSP - São Paulo (SP), Brasil.

${ }^{5}$ Doutor; Professor e Consultor do Grupo de Coluna do Departamento de Ortopedia e Traumatologia da Faculdade de Ciências Médicas da Santa Casa de São Paulo - FCMSCSP - São Paulo (SP), Brasil.

'Doutor; Professor Adjunto do Departamento de Ortopedia e Traumatologia da Faculdade de Ciências Médicas da Santa Casa de São Paulo - FCMSCSP - São Paulo (SP), Brasil.
} 
DESCRITORES: Discite;

Tuberculose da coluna vertebral/radiografia; Cifose; Antituberculosos; Doenças da coluna vertebral; Terapêutica; Tomografia computadorizada por raios-x; Imagem por ressonância magnética
KEYWORDS: Discitis;

Tuberculosis, spinal/ radiography; kyphosis;

Antitubercular agents;

Spinal diseases; Therapeutic;

Computed tomography, x-ray;

Magnetic resonance imaging
DESCRIPTORES: Discitis;

Tuberculosis de la columna vertebral/radiografía; Cifosis; Agentes antituberculosos; Enfermedades la columna vertebral; Terapéutica; Tomografía computada por Rayos X; Resonancia nuclear magnética

\section{INTRODUÇÃO}

Sir Percival Pott, em 1779, foi o primeiro autor a realizar descrição detalhada da enfermidade e sua exposição, incluindo os achados de autópsia ${ }^{1,2}$.

O termo tuberculose foi criado em 1839. É uma doença infecciosa crônica, endêmica, causada pelo Mycobacterium tuberculosis, bacilo álcool - ácido resistente, descrito por Robert Koch em 1882. Pode também ser causada por outras formas de Mycobacterium (M. bovis, M. kansasin, M. fortuitum, M. martinum, $M$. intracellulase $)^{3-6}$.

A tuberculose causa 3 milhões de mortes por ano e, somente na década de 1990, foram diagnosticados 90 milhões de $\operatorname{casos}^{7,8}$, tornando-se um dos grandes problemas de saúde pública nos países em desenvolvimento. Segundo dados da Organização Mundial de Saúde (OMS), se não houver um esforço para o controle da doença, no período de 2002 a 2020 mais de 1 bilhão de pessoas serão contaminadas e, destas, 150 milhões adoecerão, podendo, então, ocorrer 36 milhões de óbitos por tuberculose ${ }^{9}$.

Como o acometimento ósseo é responsável por $10 \%$ dos casos clínicos de tuberculose e, destes, cerca da metade é de comprometimento vertebral, os ortopedistas devem estar preparados para o tratamento de pacientes com essa doença. Porém, existem controvérsias entre os diversos autores quanto às formas de tratamento, à duração da quimioterapia e à necessidade da confirmação microbiológica da doença (achado da Mycobacterium tuberculosis no tecido ósseo) ${ }^{10-14}$.

A suspeita diagnóstica da tuberculose vertebral é difícil e, em geral, é estabelecida em estágio avançado, uma vez que as alterações características de suas lesões vertebrais são visibilizadas nas radiografias ${ }^{15}$.

$\mathrm{O}$ uso de medicamentos revolucionou o tratamento e continua sendo a base para a cura ${ }^{3}$. A intervenção cirúrgica é reservada somente para situações particulares, como associação com disfunção neurológica e instabilidade vertebral ${ }^{16}$.

O prognóstico varia de acordo com a idade dos pacientes, estado geral e imunológico, gravidade e duração do quadro neurológico e tipo de tratamento instituído, sendo que todas essas variáveis são influenciadas pelo diagnóstico precoce e correto da doença. O objetivo do tratamento da doença é curar a infecção, prevenir e tratar déficits neurológicos e prevenir e tratar as deformidades ${ }^{17-19}$.

$\mathrm{O}$ estudo avalia as características dos desfechos radiográficos dos pacientes com tuberculose óssea na coluna vertebral tratados de forma conservadora nessa instituição.

\section{MÉTODOS}

Série retrospectiva de pacientes portadores de Mal de Pott submetidos ao tratamento conservador atendidos no Grupo de Coluna do Departamento de Ortopedia e Traumatologia da Faculdade de Ciências Médicas da Santa Casa de Misericórdia de São Paulo - FCMSCSP - São Paulo (SP), com diagnóstico de tuberculose da coluna vertebral no período de janeiro de 1989 a dezembro de 2009.

Os critérios de exclusão foram: casos tratados com cirurgia devido à alteração neurológica grave (Frankel A, B e C); menos de um ano de acompanhamento; prontuário incompleto; inexistência de radiografias do início e do final do acompanhamento.

Também foi avaliada a atividade radiológica da lesão espinhal de acordo com o critério do Medical Research Council Working Party on Tuberculosis of the Spine (MRCWPTS) (Apêndice A). Esses critérios de imagem associados ao quadro clínico de inapetência, emagrecimento, febre noturna, comprometimento do estado geral sem sinais de septicemia e provas inflamatórias elevadas - Proteína C Reativa (PCR) e/ou Velocidade de Sedimentação Globular (VSG) - foram os critérios diagnósticos para a inclusão no estudo, sendo, então, empregado o teste terapêutico com esquema tríplice de poliquimioterapia por meio de: Rifampcina, Isoniazida e Pirazinamida pelo período de 12 meses com a documentação da normalidade dos valores das provas inflamatórias inespecíficas (VHS e muco proteína C).

A cifose localizada foi mensurada por meio do método de Cobb, sendo utilizadas as placas terminais preservadas imediatamente craniais e caudais ao nível acometido como referência, e a cifose regional, utilizando-se as placas terminais da vértebra superior e da vértebra inferior como referência para a medida (Figura 1). A mensuração foi realizada nas radiografias na incidência de perfil, realizadas por ocasião do diagnóstico inicial, sendo comparadas com as radiografias do final do acompanhamento, com período mínimo de segmento de um ano. Foram também colhidas informações em relação a: nível vertebral acometido, presença de alteração neurológica, sintomatologia, comorbidades e presença de tuberculose pulmonar, tipo e duração do tratamento e presença de alteração neurológica após o fim do tratamento.

Foi realizada estatística descritiva das variáveis qualitativas em termos de frequências absolutas e relativas. Para as variáveis quantitativas, foram calculadas algumas 
medidas resumo e apresentadas por meio de gráficos do tipo Boxplot $^{\circledR}$. Para comparar as variáveis quantitativas entre os instantes inicial e final utilizou-se o teste de Wilcoxon ${ }^{\circledR}$. O nível de significância obtido foi de 5\%.

\section{RESULTADOS}

Foram incluídos no trabalho 33 pacientes $(68,8 \%)$, sendo 17 pacientes $(51 \%)$ do sexo masculino e $16(48,8 \%)$ do sexo feminino (Gráfico 1). A idade variou de 3 a 87 anos, com média de 44,1 anos.

O critério para diagnóstico foi clínico e radiográfico. Os exames de imagem (radiografias, tomografia computadorizada e ressonância magnética) do segmento acometido demonstraram cifose localizada com acunhamento do corpo vertebral e lesão osteolítica da placa terminal de dois corpos vertebrais sem comprometimento do disco intervertebral associado com reação paravertebral.

O período de acompanhamento dos pacientes variou entre 12 e 329 meses, com média de 63,4 meses.

Em 12 casos as lesões poderiam ser consideradas de atividade duvidosa, segundo os critérios do MRCWPTS (Apêndice A).

Foram acometidas as vértebras torácicas $(15,1 \%)$, toracolombares $(9 \%)$ e lombares $(75,7 \%)$, sendo que nos pacientes estudados não houve acometimento da coluna cervical e lombo-sacro. $\mathrm{O}$ acometimento de uma única vértebra ocorreu em $11,1 \%$ dos pacientes, de duas vértebras em $75,6 \%$, de três vértebras em $8,9 \%$ e de cinco vértebras em $4,4 \%$.

A cifose segmentar da região acometida na coluna vertebral ocorreu na maioria dos pacientes analisados $(60,4 \%)$, e

\section{APÊNDICE A \\ CLASSIFICAÇÃO DA ATIVIDADE RADIOGRÁFICA \\ 1.Doença ativa: \\ A) rarefação dos corpos vertebrais afetados, e \\ B) perda do fino contorno cortical. \\ 2.Doença inativa (quiescente): \\ A) fusão óssea dos corpos vertebrais afetados, ou \\ B) esclerose das superfícies contíguas dos corpos vertebrais afetados com redução ou desaparecimento do espaço discal. \\ 3.Doença de atividade duvidosa: \\ A aparência da esclerose marginal onde houve tanta destruição dos corpos vertebrais que não há aposição vertebral nem acima nem abaixo do foco da doença. Nestas circunstâncias a doença é classificada como de atividade duvidosa Cavitação do corpo vertebral ou formação de sequestro não são consideradas como evidência de doença.}

Fonte: Medical Research Council Working Party on Tuberculosis of the Spine ${ }^{4}$ o aumento da angulação ocorreu em $50 \%$ deles. A média da cifose localizada antes do tratamento foi de $29,98^{\circ}$ e, após o tratamento, foi de $32,74^{\circ}$.

Observamos piora da angulação média de Cobb de 2,8 e da segmentar de $4^{\circ}$ (Tabela 1 ).

Dentre os 33pacientes $(68,8 \%)$ tratados sem cirurgia, notamos o aumento da cifotização local em 20 deles $(60,6 \%)$, e da cifose segmentar em $23(69,6 \%)$.

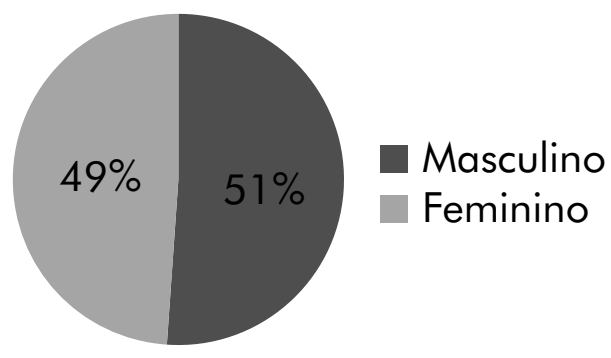

Gráfico 1

Distribuição dos portadores de Mal de Pott de acordo com o sexo

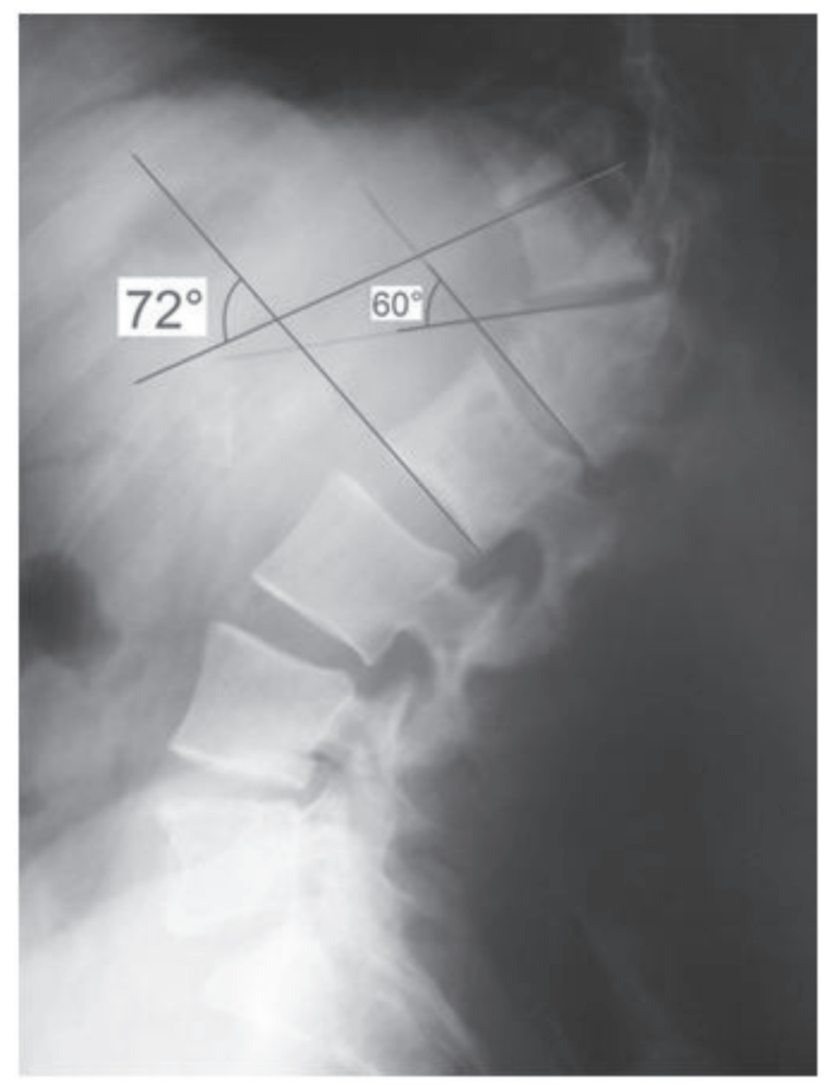

Figura 1

Ilustração da medida do ângulo de Cobb.

\section{TABELA 1 - Variação do colapso sagital}

\begin{tabular}{|c|c|c|c|c|c|c|c|}
\hline Variável & & $\mathrm{n}$ & Média & DP & Mínimo & Mediana & Máximo \\
\hline \multirow{2}{*}{ Cifose segmentar } & inicial & 33 & 29,2 & 26,4 & 0,0 & 24,0 & 105,0 \\
\hline & final & 33 & 33,21 & 27,4 & 0 & 26 & 105 \\
\hline \multirow[t]{2}{*}{ Cifose local } & inicial & 33 & 29,9 & 23,9 & 0 & 21 & 90 \\
\hline & final & 33 & 32,7 & 26 & 0 & 23 & 105 \\
\hline
\end{tabular}


Notamos diminuição da cifotização segmentar em quatro pacientes, e seis pacientes diminuíram a cifose local.

Observamos que o pior prognóstico de progressão foi o nível toracolombar $\left(11,3^{\circ}\right)$, sendo essa diferença estatisticamente significante $(\mathrm{p}<0,05)$. O nível lombar $\left(7,3^{\circ}\right)$ mostrou uma progressão maior que a torácica $\left(4,2^{\circ}\right)$, porém, essa diferença não se mostrou estatisticamente significante $(\mathrm{p}>0,05)$.

\section{DISCUSSÃO}

A tuberculose é uma doença que tem alta prevalência na população mundial e, segundo dados da OMS, estima-se que um terço dela esteja infectada pelo bacilo, ${ }^{9,20,21}$. Deste valor, apenas $15 \%$ das pessoas vão desenvolver a doença, com uma apresentação óssea em 2 a 10\% dos casos, sendo que 40 a $60 \%$ dos casos irão se localizar na coluna ${ }^{8,20-22}$.

No Mal de Pott, as lesões vertebrais, em geral, são tratadas de modo tardio, seja pelo atraso do paciente em procurar auxílio médico ou por retardo do diagnóstico ${ }^{21}$. Em nossa casuística observamos que $100 \%$ dos pacientes foram diagnosticados apresentando fase ativa da doença; portanto, estavam presentes as características radiográficas, bem como os sintomas gerais dessa enfermidade, que se instalam de forma insidiosa justificando o longo período de evolução da doença até o seu diagnóstico.

O paciente pode ou não apresentar o quadro clássico da infecção tuberculosa com perda de peso, inapetência e febre ${ }^{20,21}$. A principal queixa nos casos vertebrais é uma dorsalgia ou lombalgia de alguns meses de evolução ${ }^{23,24}$. Em nossa casuística, observamos que o quadro doloroso da coluna vertebral foi concomitante aos sintomas gerais da doença, provavelmente pelo período longo de evolução da infecção até o seu diagnóstico.

$\mathrm{Na}$ série apresentada, a dorsalgia e a lombalgia foram as principais queixas (100\%). O déficit motor de 41,9\% está dentro dos padrões relatados pela maioria dos autores $(12,5 \text { a } 64,29 \%)^{21,23,25}$, mas bem superior aos relatos do MRCWPTS.

$\mathrm{Na}$ literatura, um exame importante para auxiliar o diagnóstico é o teste de Mantoux ${ }^{\circledR}$, que indica contato com as micobactérias. Contudo, como a vacinação com BCG faz parte do programa básico de vacinas do Ministério da Saúde, o teste perde parcialmente o valor em nosso país, uma vez que indivíduos imunizados podem apresentar teste cutâneo positivo devido à reação imunológica ativa proporcionada pela vacinação ${ }^{7,26}$. Esse fato adicionado à baixa especificidade e reprodutibilidade do método explicam porque tal estudo diagnóstico não foi critério de inclusão no nosso estudo. A radiografia de tórax é outro exame auxiliar para o diagnóstico, sendo positiva de um terço à metade dos pacientes do estudo. Porém, revela somente a imagem característica do primeiro contato com o bacilo ou, nas formas pulmonares de tuberculose, revela o acometimento mais disseminado. No entanto, a literatura confirma que é eventual o acometimento pulmonar e extrapulmonar concomitante; assim, esse exame também não representa critério objetivo para diagnóstico de Mal de Pott ${ }^{10,23,25}$. Nesta casuística, encontramos apenas cinco casos positivos, com tuberculose miliar. O padrão radiográfico de acometimento vertebral pode ocorrer de três formas: lesão vertebral com comprometimento discal (lesão clássica), lesão vertebral isolada (sem comprometimento discal) ou acometimento de elementos posteriores (difícil visibilização nas radiografias simples) ${ }^{25}$. Apresentaram lesão clássica $75 \%$ dos pacientes e lesão vertebral isolada os outros $25 \%$.

Muitos autores utilizam a biópsia como exame confirmatório da doença. ${ }^{23,25,26} \mathrm{Em}$ nosso serviço, não é rotina a realização de biópsia vertebral nos casos suspeitos de tuberculose pela baixa positividade e pelo fato de muitos autores não a considerarem fundamental para o tratamento ${ }^{12,20,27}$. Outros autores nacionais adotam a conduta do diagnóstico clínico-radiográfico, com baixa frequência de biópsias pelo alto índice de achados inespecíficos e de necrose ${ }^{28,29}$.

O diagnóstico de certeza só pode ser confirmado com o achado do bacilo; porém, o nosso serviço adota a orientação de tratar todas as lesões com diagnóstico de probabilidade (lesão clássica à radiografia), válido para regiões com alta prevalência de tuberculose na população ${ }^{12,13,20,21}$. Entretanto, apenas o critério radiológico tem suas falhas, pois outras infecções, como a brucelose, apresentam quadro radiológico similar, o que constituiu uma falha na triagem de um paciente nesta série.

Foram utilizados como auxiliares do diagnóstico de infecção os exames laboratoriais. O controle seriado do VSG e PCR são citados para avaliar o sucesso da quimioterapia ${ }^{14,20}$, o que ocorreu também com os pacientes da série apresentada, observando melhora do padrão radiológico com diminuição do VSG e PCR. Por isso, o teste terapêutico com o esquema tríplice é realizado no nosso serviço.

É consenso na literatura o tratamento específico com esquemas múltiplos, e a dificuldade está em estabelecer a duração da quimioterapia ${ }^{10,14,25}$. Inicialmente, o tratamento quimioterápico ideal era de 30 meses $^{25}$. Atualmente, grande parte dos autores preconiza esquemas entre 12 e 18 meses de duração ${ }^{12-14,20,22,30}$. Existem orientações mais recentes para a diminuição do tempo de quimioterapia (seis meses), facilitando a adesão do paciente e apresentando eficácia comparável a regimes mais longos. A orientação do Ministério da Saúde ${ }^{7}$ em nosso meio prevê um esquema de seis meses para os casos de tuberculose extrapulmonar, virgem de tratamento. Concordamos com os autores que preconizam pelo menos um ano de tratamento com monitoração por meio de provas inflamatórias (hemograma, VSG e PCR).

A imobilização externa foi utilizada como tratamento incruento até que existissem sinais clínicos e radiológicos de cura da doença, conduta adotada por outros autores nacionais $^{30,31}$. Tem sido demonstrado por alguns autores que o uso prolongado da imobilização não altera a taxa de fusão ou deformidade residual, recomendando seu uso nos casos de dor ou espasmo muscular ${ }^{13,27,31}$.

Não existe uniformidade em relação à correção da cifose nem de quanto seria a medida a partir da qual se 
indica correção, sendo que existe uma tendência para indicação em torno de $30 \square$ de cifose ${ }^{11,12,22}$. Apesar de muito citada pela literatura, a fórmula clássica de Rajasekaran e Shanmugasundaram, a qual prevê a cifose final em $90 \%$ dos casos e só é aplicável para as lesões acima de L2, não é utilizada pela maioria dos autores que aguardam a evolução para indicar o tratamento cirúrgico. Nos pacientes do estudo não foi indicada correção apenas por queixa estética, dor ou deformidade angular.

Paciente de 45 anos, do sexo feminino, apresentou, no diagnóstico inicial da doença, quadro de febre, anorexia, perda de peso, tosse, febre, dor lombar e sintomas neurológicos nos membros inferiores (Frankel D).

A paciente foi tratada conservadoramente com antibioticoterapia e colete TLSO por nove meses e evoluiu com melhora do quadro neurológico (Frankel E) (Figuras 2, 3 e 4).

\section{CONCLUSÃO}

Nesta série de casos de tuberculose vertebral tratados de modo conservador, a evolução radiológica foi satisfatória.



Figura 3

Pré-tratamento.

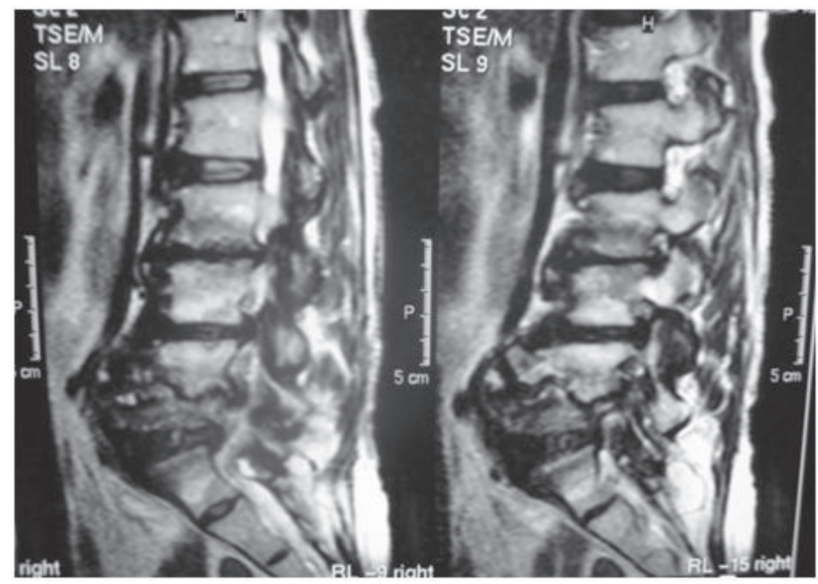

Figura 2

Pré-tratamento.
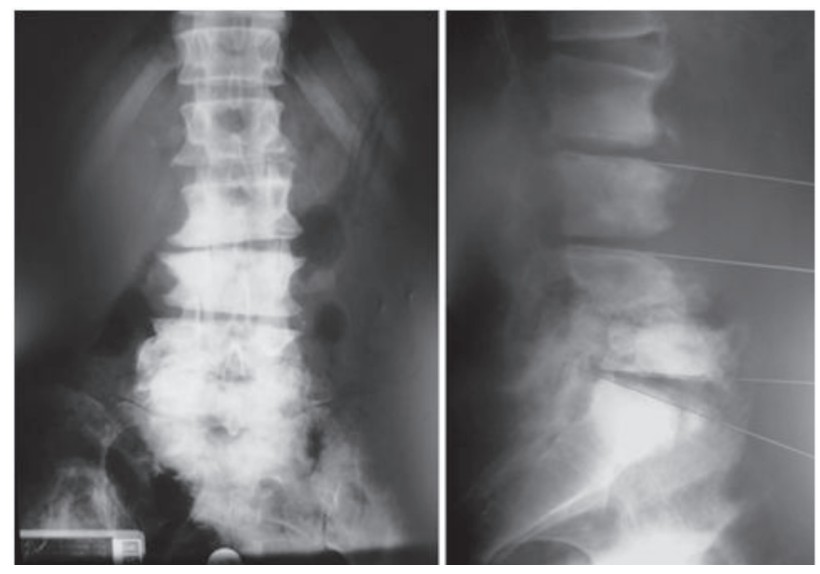

Figura 4

Pós-tratamento.

\section{REFERÊNCIAS}

1. Hippocrates. The genuine works of Hippocrates, translated by F. Adams. London: The Sydenham Society; 1849.

2. Pott P. Remarks on that kind of palsy of the lower limbs is frequently found to accompany a curvature of the spine. London: J. Johnson; 1779.

3. Albee $\mathrm{CH}$, Powers EJ, McDowell HC. Tuberculoses of the spine; surgery of the spinal column. Philadelphia: F.A. Davis; 1945. Chapter VI, p. 150-225.

4. Carnelase PG. Tuberculose. In: Crenshaw AH, editor. Cirurgia ortopédica de Campbell; 1989. cap. 29, p. 727-37.

5. Chu CB. Treatment of spinal tuberculosis in Korea, using focal débridement and interbody fusion. Clin Orthop Relat Res. 1967;(50):235-53.
6. Rafful M. Tuberculose pulmonar. In: Silveira IC. O Pulmão. Rio de Janeiro: Publicações Médicas; 1987. cap. 21, p. 223-34.

7. Brasil. Ministério da Saúde. Fundação Nacional de Saúde. Centro de Referência Prof. Hélio Fraga. Sociedade Brasileira de Pneumologia e Tisiologia. Controle da tuberculose: uma proposta de integração ensino-serviço. 5a ed. Rio de Janeiro: FUNASA/CRPHF/SBPT; 2002.

8. Mehta JS, Bhojraj SY. Tuberculosis of the thoracic spine. A classification based on the selection of surgical strategies. J Bone Joint Surg Br. 2001;83(6):859-63.
9. World Health Organization. Tuberculosis: Fact Sheet No.104 Revised April 2005 [ Internet]. Genebra: WHO; 2006. [cited 2005 Jun 12]. Available from: http:// www. who.int/mediacentre/factsheets/ who104/en.

10.A controlled trial of ambulant outpatient treatment and in-patient rest in bed in the management of tuberculosis of the spine in young Korean patients on standard chemotherapy a study in Masan, Korea. First report of the Medical Research Council Working Party on Tuberculosis of the Spine. J Bone Joint Surg Br. 1973;55(4):67897.

11.Moon MS, Woo YK, Lee KS, Ha KY, Kim SS, Sun DH. Posterior instrumentation and anterior interbody fusion for tuberculous kyphosis of dorsal and lumbar spines. Spine (Phila Pa 1976). 1995;20(17):1910-6. 
12.Al-Sebai MW, Al-Khawashki H, Al-Arabi K, Khan F. Operative treatment of progressive deformity in spinal tuberculosis. Int Orthop. 2001;25(5):322-5.

13. Moon MS, Kim I, Woo YK, Park YO. Conservative treatment of tuberculosis of the thoracic and lumbar spine in adults and children. Int Orthop. 1987;11(4):315-22.

14.Moon MS, Moon YW, Moon JL, Kim SS, Sun DH. Conservative treatment of tuberculosis of the lumbar and lumbosacral spine. Clin Orthop Relat Res. 2002;(398):40-9

15.Teo EL, Peh WC. Imaging of tuberculosis of the spine. Singapore Med J. 2004;45(9):439-44.

16. Martin NS. Tuberculosis of the spine. A study of the results of treatment during the last twenty-five years. J Bone Joint Surg Br. 1970;52(4):61328.

17.Ho EK, Leong JC. Tuberculosis of the spine. In: Weinstein SL, editor. The Pediatric Spine: Principles and Practice. New York: Raven Press; 1994.

18.Tuli S. Tuberculosis of the Skeletal System. Jaypee Brothers Medical; 1991.
19.Oguz E, Sehirlioglu A, Altinmakas M. Ozturk C, Komurcu M, Solakoglu $\mathrm{C}$, et al. A new classification and guide for surgical treatment of spinal tuberculosis. Int Orthop. 2008;32(1):127-33.

20.Bhojraj S, Nene A. Lumbar and lumbosacral tuberculous spondylodiscitis in adults. Redefining the indications for surgery. J Bone Joint Surg Br . 2002;84(4):530-4.

21.Moore SL, Rafii M. Imaging of musculoskeletal and spinal tuberculosis. Radiol Clin North Am. 2001;39(2):329-42.

22.Tuli SM. Severe kyphotic deformity in tuberculosis of the spine. Int Orthop. 1995;19(5):327-31.

23.Garcia-Lechuz JM, Julve R, Alcala L, Ruiz-Serrano MJ, Muñoz P. Espondilodiscitis tuberculosa o enfermedad de Pott: experiência en un hospital general. Enferm Infecc Microbiol Clin. 2002;20(1):5-9.

24.Caksen H, Uzüm K, Tutus A. Pott's disease. Clin Nucl Med. 2001;26(1):57.

25.Friedman B. Chemotherapy of tuberculosis of the spine. J Bone Joint Surg Am. 1966;48(3):451-74

26.Moore SL, Rafii M. Imaging of musculoskeletal and spinal tuberculosis. Radiol Clin North Am. 2001;39(2):329-42.

27.Bewes P. Spinal tuberculosis. Trop Doct. 2001;31(4):237-40.
28.Puertas EB, Chagas JCM, Wajchenberg M. Avaliação clinica radiológica e tratamento de 17 pacientes com tuberculose óssea na coluna vertebral. Rev Bras Ortop. 1999;34(2):113-6.

29.Mandetta H, Pereira FA, Sismeiro LFF. Mal de Pott: tratamento clínico e cirúrgico. Rev Bras Ortop. 1994;29(3):139-43.

30.A 10-year assessment of controlled trials of inpatient and outpatient treatment and of plaster-of-Paris jackets for tuberculosis of the spine in children on standard chemotherapy. Studies in Masan and Pusan, Korea. J Bone Joint Surg Br. 1985;67(1):10310.

31. Schulitz KP, Kothe R, Leong JC, Wehling P. Growth changes of solidly fused kyphotic bloc after surgery for tuberculosis. Comparison of four procedures. Spine (Phila Pa 1976). 1997;22(10):1150-5.

\section{Correspondência}

Carlos Humberto Targa Moreira

Instituto de Ortopedia, Traumatologia e Reabilitação de Dourados - MS (ITOR)

Rua João Rosa Góes, 830

CEP 79804-020 - Dourados (MS) Brasil

E-mail: itor-carloshumberto@hotmail.com 